\title{
Dynamic changes in serum IL-6, IL-8, and IL-10 predict the outcome of ICU patients with severe COVID-19
}

\author{
Jia Li ${ }^{1 \#}$, Liu Rong ${ }^{2 \#}$, Ran Cui ${ }^{3}$, Jiaqi Feng ${ }^{1}$, Yuyang Jin ${ }^{1}$, Xiaoxiang Chen ${ }^{1}$, Renying Xu ${ }^{4}$ \\ ${ }^{1}$ Department of Rheumatology, Renji Hospital, Shanghai Jiao Tong University School of Medicine, Shanghai, China; ${ }^{2}$ Department of Critical Care \\ Medicine, Shanghai Jiao Tong University School of Medicine Affiliated Shanghai General Hospital, Shanghai, China; ${ }^{3}$ Department of Rheumatology \\ and Immunology, Shanghai Jiao Tong University School of Medicine Affiliated Six People's Hospital, Shanghai, China; ${ }^{4}$ Department of Clinical \\ Nutrition, Renji Hospital, Shanghai Jiao Tong University School of Medicine, Shanghai, China \\ Contributions: (I) Conception and design: J Li, L Rong, X Chen, R Xu; (II) Administrative support: L Rong, R Cui, X Chen; (III) Provision of study \\ materials or patients: J Li, L Rong, R Cui, J Feng, Y Jin; (IV) Collection and assembly of data: J Li, L Rong, R Cui, J Feng, Y Jin; (V) Data analysis \\ and interpretation: J Li, L Rong, X Chen, R Xu; (VI) Manuscript writing: All authors; (VII) Final approval of manuscript: All authors. \\ \#These authors contributed equally to this work. \\ Correspondence to: Renying Xu, MD, PhD; Xiaoxiang Chen, MD, PhD. 145 Middle Shandong Road, Shanghai 200001, China. \\ Email: Xurenying7465@126.com; Xiaoxiang0721@126.com.
}

\begin{abstract}
Background: Biomarkers to prognosticate the outcomes and guide the treatment of patients with severe coronavirus disease 2019 (COVID-19) are currently required. We aimed to investigate whether the dynamic variation of cytokines was associated with the survival of patients admitted to the intensive care unit (ICU).

Methods: A retrospective study was performed on 40 patients with COVID-19 admitted to the ICU in Wuhan, China. Demographic, clinical, and laboratory variables were collected, and serum cytokines were kinetically assessed. A multivariable-adjusted generalized linear regression model was used to analyze the differences in serum cytokine levels between survivors and non-survivors.

Results: Among the 40 patients included, we found a positive correlation between multiple cytokines. Serum levels of interleukin (IL)-6, IL-10, and tumor necrosis factor alpha (TNF $\alpha$ ) in non-survivors were consistently elevated compared to those in the survivors. Kinetic variations in IL-6, IL-8, and IL-10 were associated with a fatal outcome in patients with severe COVID-19, independent of sex, age, absolute lymphocyte count, direct bilirubin, hypertension, chronic obstructive pulmonary disease, and cancer as well as the use of glucocorticoids and tocilizumab.
\end{abstract}

Conclusions: Dynamic changes in serum IL-6, IL-8, and IL-10 levels were associated with survival in patients in the ICU, and could serve as a predictive biomarker to determine the therapeutic options for patients with severe COVID-19.

Keywords: Coronavirus disease 2019 (COVID-19); cytokines; interleukin-6 (IL-6); IL-8; IL-10

Submitted Oct 28, 2020. Accepted for publication Jan 08, 2021.

doi: 10.21037/apm-20-2134

View this article at: http://dx.doi.org/10.21037/apm-20-2134

\section{Introduction}

The coronavirus disease 2019 (COVID-19) pandemic has led to more than 38 million infections and 1 million deaths worldwide. The pathogenesis and effective treatments for the infection caused by the severe acute respiratory syndrome coronavirus 2 (SARS-CoV-2) remain to be fully understood. The survival rate of patients with severe COVID-19 in the intensive care unit (ICU) is much lower than that of patients with mild COVID-19. Hyperinflammation and pro-inflammatory cytokines are key factors in the clinical course of acute respiratory distress syndrome and multiple organ dysfunction syndrome in severe patients with COVID-19 (1). Studies 
have demonstrated a correlation between the clinical and laboratory characteristics, and the outcomes in patients with COVID-19 (2,3). Clinical trials performed on patients with COVID-19, focusing on targeting cytokine signals, including interleukin (IL)-6, IL-1, granulocyte-macrophage colony-stimulating factor antagonists, or small-molecule compounds, have reported both benefits and failure (4-10). Serum cytokine profiles have been shown to predict the severity of COVID-19 $(11,12)$. However, little is known about the correlation between the dynamics of serum cytokine alterations and different prognoses of patients with severe COVID-19 during hospitalization. More evidence is thus needed to determine whether cytokine profiles are reliable biomarkers for inflammation processes and predict the outcome of severely ill patients with heterogeneity (13). In this retrospective study, we explored the relationship between the kinetic variations of cytokines and the clinical outcomes in patients with severe COVID-19 admitted to the ICU. We present the following article in accordance with the STROBE reporting checklist (available at http:// dx.doi.org/10.21037/apm-20-2134).

\section{Methods}

\section{Study design and participants}

This retrospective cohort single-center study included 40 adult patients with COVID-19 who were admitted to the ICU at the Wuhan Leishenshan Hospital in China, from February 19 to April 6, 2020. Wuhan Leishenshan Hospital was an emergency specialty field hospital for patients with COVID-19 and was managed by Zhongnan Hospital of Wuhan University and a medical team from Shanghai. COVID-19 was diagnosed based on the presence of clinical symptoms and SARS-CoV-2 nucleic acid using a real-time reverse transcription polymerase chain reaction assay with nasal and pharyngeal swab specimens. All the individuals with COVID-19 who were admitted to the ICU, according to the Chinese Guidelines for the management of COVID-19 (Trial Version 6) (14), were enrolled in this study. The study was performed in accordance with the Declaration of Helsinki (as revised in 2013) and approved by the Ethics Committee of Shanghai General Hospital of Shanghai Jiao Tong University School of Medicine (No. 2020-29). Individual consent was waived for this retrospective study.

\section{Data collection}

Demographic, clinical, and laboratory parameters, including cytokine test, and outcome data of the ICU patients were obtained from electronic medical records; the data were checked by two physicians. The normal concentration ranges for IL-1 $\beta$, IL-8, IL-10, and tumor necrosis factor alpha (TNF $\alpha$ ) were below 5, 62, 9.1, and $8.1 \mathrm{pg} / \mathrm{mL}$, respectively. The normal concentration range of IL-6 was between $0-7 \mathrm{pg} / \mathrm{mL}$ and that of normal IL-2 receptor (IL-2R) ranged from 223 to $710 \mathrm{U} / \mathrm{mL}$. The sera of patients were repeatedly analyzed and further classified into four groups ( $\leq 3,4-7,8-13$, and $\geq 14 \mathrm{~d}$ ) according to the length of stay in the ICU to understand the dynamic variation in cytokines.

\section{Statistical analysis}

We performed all the statistical analyses using SAS version 9.4 (SAS Institute, Inc, Cary, USA). The formal hypothesis testing performed was two sided with a significance level of $\mathrm{P}<0.05$. Classification variables were expressed as percentage, and the differences between two groups were tested using the Chi-square test. The quantified variables were expressed as mean \pm standard deviation, and the differences were analyzed using the Student's $t$-test for normal distribution, whereas the data were expressed as median and interquartile range (IQR) for abnormal distribution, and the differences were analyzed using the Kruskal-Wallis $\mathrm{H}$ test. We used a multivariableadjusted generalized linear regression model to evaluate the differences in serum cytokine levels between survivor and non-survivor patients after adjusting for the covariates that were selected using univariate analysis. Figures were generated using GraphPad Prism version 8.0 (GraphPad Software, San Diego, California, USA).

\section{Results}

\section{Demographic and baseline clinical characteristics of patients with COVID-19}

A total of 40 patients with severe COVID-19 admitted to the ICU were included in this study, who were referred to the ICU department from general wards. The patients were prescribed traditional Chinese medicine and symptomatic treatment prior to ICU admission. The median age was 71 years (IQR $61-81$ years) and $60 \%$ of the sample population comprised men. Fever (55\%), cough (55\%), anorexia or diarrhea (45\%), dyspnea (32.5\%), and fatigue $(32.5 \%)$ were the commonly reported symptoms in the patients. Comorbidity of malignancy $(7.5 \%)$, hypertension 
Table 1 Demographics and baseline laboratory characteristics of patients with COVID-19 in the ICU

\begin{tabular}{|c|c|c|}
\hline Characteristics & $\begin{array}{l}\text { Reference } \\
\text { range }\end{array}$ & Parameters \\
\hline \multicolumn{3}{|l|}{ Demographic } \\
\hline Age (years) & & 71 [61-81] \\
\hline Gender (male) & & $24(60 \%)$ \\
\hline $\begin{array}{l}\text { Days from onset of } \\
\text { symptoms to admission to } \\
\text { ICU }\end{array}$ & & 20 [11-29] \\
\hline Days in ICU & & $8.5[5.25-15.75]$ \\
\hline \multicolumn{3}{|l|}{ Comorbidity } \\
\hline Diabetes & & $10(25 \%)$ \\
\hline Malignancy & & $3(7.5 \%)$ \\
\hline Hypertension & & $19(47.5 \%)$ \\
\hline $\begin{array}{l}\text { Chronic obstructive } \\
\text { pulmonary disease }\end{array}$ & & $4(10 \%)$ \\
\hline Secondary bacteria infection & & $5(12.5 \%)$ \\
\hline \multicolumn{3}{|l|}{ Laboratory characteristics } \\
\hline $\begin{array}{l}\text { Absolute neutrophil count } \\
\text { (per } \mathrm{mL} \text { ) }\end{array}$ & $1,800-6,300$ & $5,900[3,600-8,700]$ \\
\hline $\begin{array}{l}\text { Absolute lymphocyte count } \\
\text { (per } \mathrm{mL} \text { ) }\end{array}$ & $1,100-3,200$ & $810[600-1,300]$ \\
\hline Total bilirubin (mmol/L) & $2-26$ & $10[6.8-16]$ \\
\hline Lactate dehydrogenase (U/L) & $125-243$ & 264 [217-387] \\
\hline Blood urea nitrogen (mmol/L) & $2.8-7.6$ & $7.9[5.2-14]$ \\
\hline D-dimer (mg/L) & $0-0.55$ & $2.5[1.4-4.4]$ \\
\hline C-reactive protein (mg/L) & $0-4$ & 27 [9-54] \\
\hline
\end{tabular}

Data are expressed as median [interquartile range], or $n(\%)$. COVID-19, coronavirus disease 2019; ICU, intensive care unit.

(47.5\%), diabetes mellitus (25\%), and chronic obstructive pulmonary disease $(10 \%)$ were additionally reported. Twenty-two patients $(57.5 \%)$ were treated using oxygen therapy, including nasal cannula and mask oxygen. Additionally, high-flow oxygen and non-invasive ventilation $(22.5 \%)$, tracheal intubation $(20 \%)$, and extracorporeal membrane oxygenation $(n=2)$ were performed on the patients. The median period from the time of onset of symptoms to admission into ICU was 20 days (IQR 11-29 days), and the median time of ICU stay was 8.5 days (IQR 5.25-15.75 days). ICU hospitalization of 13
$(32.5 \%)$ patients was longer than 2 weeks. From the sample population, $27.5 \%$ patients died in the ICU and $72.5 \%$ patients were discharged. The patients were administered antiviral agents (57.5\%) and/or antibiotics (92.5\%). Nineteen patients $(47.5 \%)$ underwent corticosteroid therapy. More specifically, intravenous dexamethasone (5 mg) or methylprednisolone $(40 \mathrm{mg}$ ) daily were initially used and tampered based on the patients' condition. Three patients $(7.5 \%)$ received tocilizumab due to critical illness and all of them were in non-survivors. Three patients (7.5\%) showed a consistently positive test for SARS-CoV-2 nucleic acid who died during their stay in the ICU. Bacteria were identified in five patients (12.5\%) which consisted of three blood cultures with Staphylococcus aureus, Klebsiella pneumoniae or Acinetobacter baumannii, three sputum cultures with Acinetobacter baumannii, two urine cultures with Enterococcus faecium. Then antibiotic drugs were used based on the drug sensitivity test. All of five patients were among those survivors. Additional details have been summarized in Table 1.

\section{Correlation between the time-dependent expression of cytokines and patient outcomes}

We analyzed the correlation between cytokine levels and explored the effect of longitudinal change of cytokines on the outcomes of severely ill patients in the ICU. The concentrations of serum cytokines, including IL-1 $\beta, \mathrm{IL}-2 \mathrm{R}$, IL-6, IL-8, IL-10, and TNF $\alpha$, were measured at different times since the patients were admitted to the ICU (Table 2).

Among them, IL-1 $\beta$ was within the normal range in almost all the patients; this was in agreement with the previous studies. We analyzed the correlation between six types of cytokines and found a positive correlation (Table 3). IL-2R had the highest correlation with TNF $(\mathrm{r}=0.69652$, $\mathrm{P}<0.0001)$ and IL-6 showed a strong correlation with IL-10 ( $\mathrm{r}=0.63088, \mathrm{P}<0.0001)$. The dynamic changes in cytokine profiles of survivors and non-survivors have been illustrated in Figure 1. The serum levels of IL-6, IL-10, IL8 , and TNF $\alpha$ in non-survivors showed a gradual increase compared with that in the survivors during their stay in the ICU. Upon admission to the ICU, the serum levels of IL-6 were significantly high in non-survivors. The serum levels of IL-10 were statistically different between survivors and non-survivors after three days committed to ICU. IL-8 serum levels were statistically different in two weeks of hospitalization $(\mathrm{P}<0.05)$. 


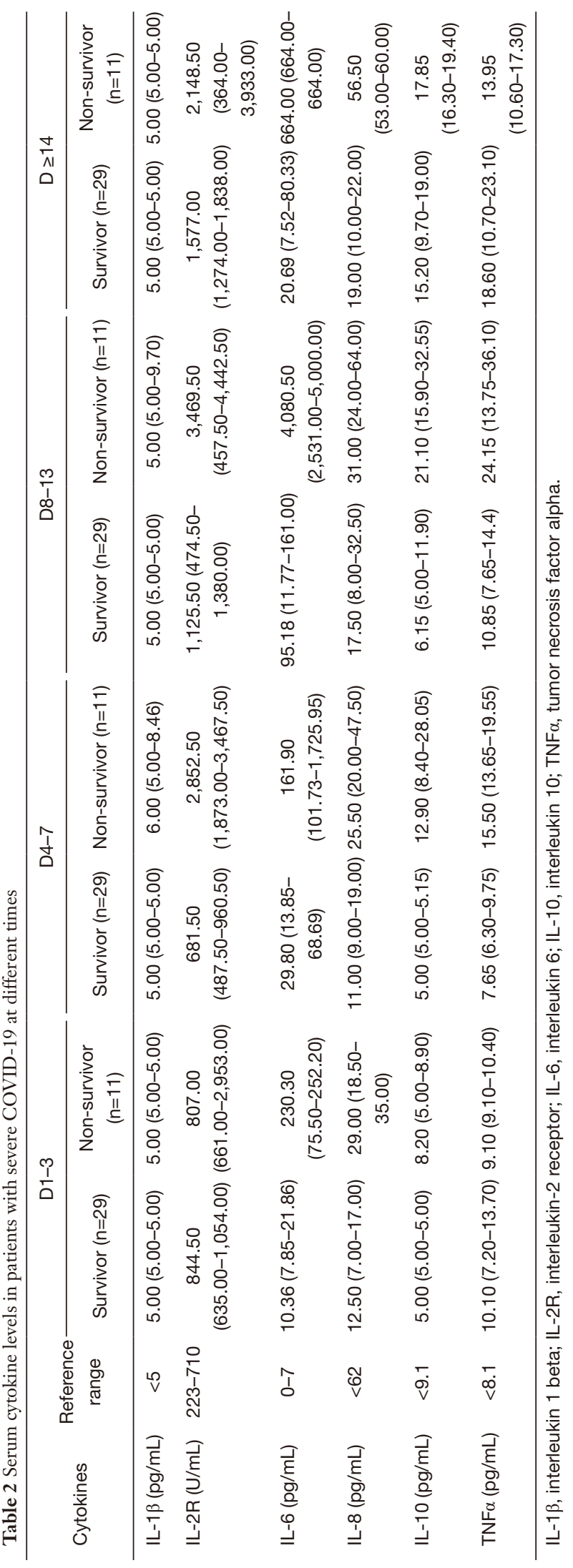

\section{$I L-6, I L-8$, and $I L-10$ are associated with the survival of patients with severe COVID-19 admitted to the ICU}

To further explore the role of cytokines in the outcome of severe patients with COVID-19, we used a generalized linear regression model to analyze the differences in serum cytokine levels between two outcome groups (survivor $v s$. non-survivor). The following factors were considered for analysis: age, gender, absolute neutrophil count, absolute lymphocyte count, direct bilirubin, lactate dehydrogenase, blood urea nitrogen, C-reactive protein, D-dimer, and the use of glucocorticoids and tocilizumab as well as the history of hypertension, diabetes mellitus, chronic obstructive pulmonary disease, and cancer (15). By using univariate analysis, we found that age $(\mathrm{P}<0.001)$, absolute lymphocyte count $(\mathrm{P}=0.005)$, direct bilirubin $(\mathrm{P}<0.001)$, history of hypertension $(\mathrm{P}<0.001)$, chronic obstructive pulmonary disease $(\mathrm{P}=0.002)$, cancer $(\mathrm{P}<0.001)$ and tocilizumab use $(\mathrm{P}=0.017)$ were associated with the outcome (Table 4 and Table S1).

We further found that IL-6, IL-8, and IL-10 were associated with the outcome of patients with severe COVID-19 after factoring sex, age, lymphocyte count, direct bilirubin, history of chronic obstructive pulmonary disease, cancer, and hypertension as well as the use of glucocorticoids and tocilizumab (Table 5).

\section{Discussion}

The prevalence of COVID-19 and corresponding high fatality in the ICU are major problems. Finding an ideal therapeutic strategy for patients in the ICU has been a challenge under the current circumstances. Identifying biomarkers that are produced during the disease course would thus be helpful for guiding treatment and supervising responses. Cytokine storm syndrome or hyperinflammation are involved in the pathogenesis of COVID-19. Several studies have shown that different inflammatory cytokines correlate with the disease severity and patient survival $(16,17)$. This study provides evidence that the kinetic variation of IL-6, IL-8, and IL-10 was closely associated with the prognosis in patients with severe COVID-19 independent of other risk factors including demographics, comorbidities, and common laboratory markers.

IL-6 is a potent predictive biomarker in patients with COVID-19 $(11,18)$. IL-6, produced by multiple cell types, including monocytes and macrophages is involved in inflammation and immune response and plays a role in the 
Table 3 Correlation analysis between circulating levels of cytokines in patients with severe COVID-19

\begin{tabular}{|c|c|c|c|c|c|c|}
\hline Cytokine & IL-1 $\beta$ & IL-2R & IL-6 & IL-8 & IL-10 & TNF $\alpha$ \\
\hline$P$ value & & 0.0965 & 0.4773 & 0.0194 & 0.0036 & 0.0007 \\
\hline IL-2R & 0.21655 & 1 & 0.31517 & 0.46515 & 0.38548 & 0.69652 \\
\hline$P$ value & 0.0965 & & 0.0142 & 0.0002 & 0.0024 & $<0.0001$ \\
\hline$P$ value & 0.4773 & 0.0142 & & 0.0003 & $<0.0001$ & 0.0019 \\
\hline IL-8 & 0.30113 & 0.46515 & 0.4547 & 1 & 0.31438 & 0.59501 \\
\hline$P$ value & 0.0194 & 0.0002 & 0.0003 & & 0.0144 & $<0.0001$ \\
\hline IL-10 & 0.36974 & 0.38548 & 0.63088 & 0.31438 & 1 & 0.52021 \\
\hline$P$ value & 0.0007 & $<0.0001$ & 0.0019 & $<0.0001$ & $<0.0001$ & \\
\hline
\end{tabular}

IL-1 $\beta$, interleukin 1 beta; IL-2R, interleukin-2 receptor; IL-6, interleukin 6; IL-10, interleukin 10; TNF $\alpha$, tumor necrosis factor alpha.

IL-1 $\beta$

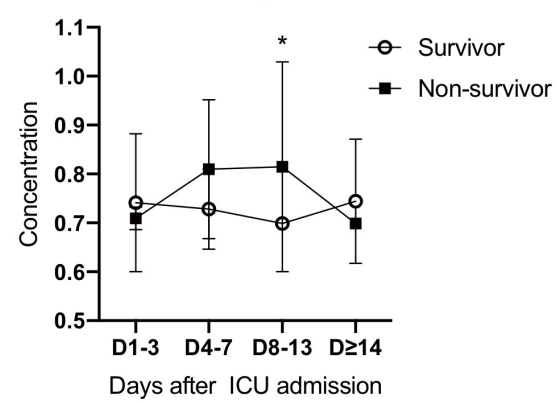

IL-8

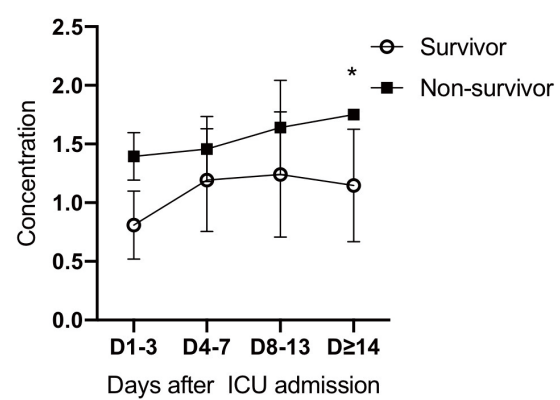

IL-2R

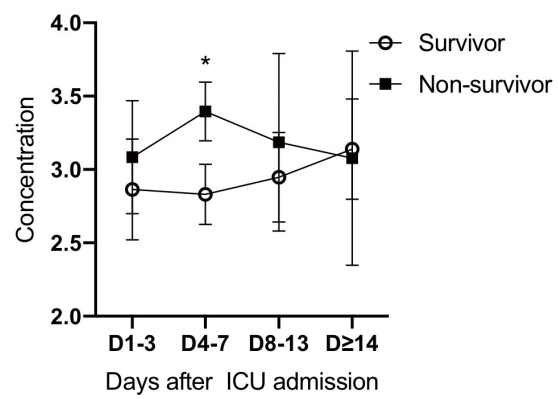

IL-10

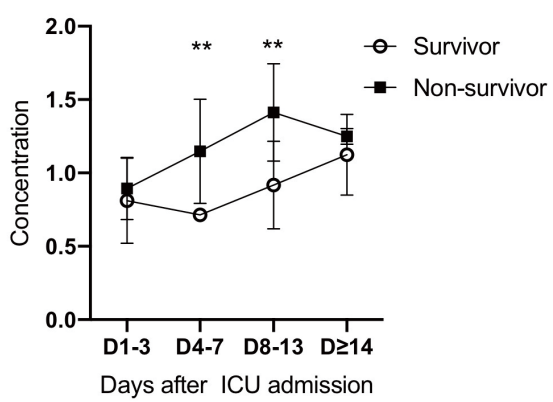

IL-6

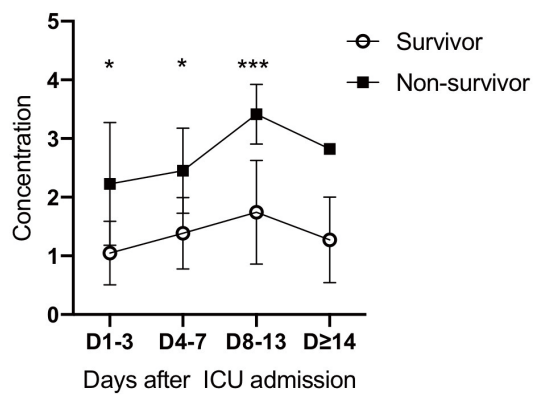

TNF- $\alpha$

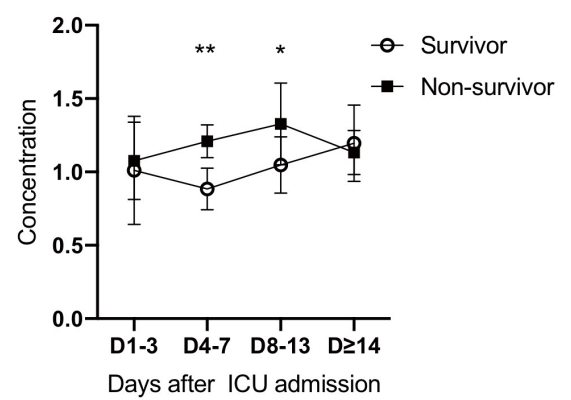

Figure 1 Dynamic changes in cytokine profiles of patients with severe COVID-19. The concentrations of circulating IL-1 $\beta$, IL-2R, IL6, IL-8, IL-10, and TNF $\alpha$ were analyzed at different time points after admission to the ICU. Data are represented as mean \pm SD. The significant differences between survivors and non-survivors were compared using the Kruskal-Wallis $\mathrm{H}$ test and are indicated as *, $\mathrm{P}<0.05$; **, $\mathrm{P}<0.01$; and ***, $\mathrm{P}<0.001$. This figure was generated using GraphPad Prism version 8.0. IL-1 $\beta$, interleukin 1 beta; IL-2R, interleukin-2 receptor; IL-6, interleukin 6; IL-10, interleukin 10; TNF $\alpha$, tumor necrosis factor alpha. 
Table 4 Demographics and baseline laboratory characteristics of patients in ICU with different outcomes

\begin{tabular}{|c|c|c|c|c|}
\hline Characteristics & Reference range & Survivor ( $\mathrm{n}=29$ ) & Non-survivor $(n=11)$ & $P$ value \\
\hline Age (years) & & 62 [72-83] & $61[47-73]$ & $<0.001$ \\
\hline Gender (male) & & $19(65.5 \%)$ & $5(45.5 \%)$ & 0.13 \\
\hline \multicolumn{5}{|l|}{ Comorbidity } \\
\hline Malignancy & & $1 / 29(3.4 \%)$ & $2 / 11(18.1 \%)$ & $<0.001$ \\
\hline Hypertension & & $15 / 29(51.7 \%)$ & 4/11 (36.4\%) & $<0.001$ \\
\hline $\begin{array}{l}\text { Chronic obstructive pulmonary } \\
\text { disease }\end{array}$ & & $3 / 29(10.3 \%)$ & $1 / 11(9.1 \%)$ & 0.002 \\
\hline Direct bilirubin ( $\mu \mathrm{mol} / \mathrm{L})$ & $0-7$ & $3.60[2.60-5.95]$ & $9.95[5.80-19.15]$ & $<0.001$ \\
\hline Blood urea nitrogen (mmol/L) & $2.8-7.6$ & $7.00[4.85-13.15]$ & $9.95[5.80-19.15]$ & 0.32 \\
\hline Lactate dehydrogenase (U/L) & $125-243$ & 259.5 [214.3-348] & 347 [235.5-477.5] & 0.39 \\
\hline D-dimer (mg/L) & $0-0.55$ & $2.12[1.17-3.86]$ & 3.06 [1.43-7.49] & 0.16 \\
\hline C-reactive protein (mg/L) & $0-4$ & $22.66[5.98-42]$ & 138 [87.9-138.0] & 0.16 \\
\hline \multicolumn{5}{|l|}{ Treatment } \\
\hline Glucocorticoid & & $13 / 29(44.8 \%)$ & $6 / 11(54.5 \%)$ & 0.583 \\
\hline
\end{tabular}

Table 5 Mean differences and $95 \%$ confidence intervals for changes in six cytokines according to the outcome groups

\begin{tabular}{lccc}
\hline Cytokines & Survivors $(n=29)$ & Non-survivors $(\mathrm{n}=11)$ & $P$ value \\
\hline IL-1 $\beta$ & Ref $(0)$ & $0.03(-0.04,0.10)$ & 0.42 \\
IL-2R & Ref $(0)$ & $0.07(-0.18,0.32)$ & 0.57 \\
IL-6 & $\operatorname{Ref}(0)$ & $1.28(0.73,1.83)$ & $<0.001$ \\
IL-8 & Ref $(0)$ & $0.29(0.03,0.55)$ & 0.03 \\
IL-10 & Ref $(0)$ & $0.23(0.03,0.43)$ & 0.02 \\
TNF $\alpha$ & $\operatorname{Ref}(0)$ & $0.09(-0.08,0.27)$ & 0.28 \\
\hline
\end{tabular}

(I) All the cytokines were log-transformed. (II) The model was adjusted for sex, age (y), lymphocyte (/mL), direct bilirubin (mmol/L), glucocorticoids (yes vs. no), tocilizumab (yes vs. no), chronic obstructive pulmonary disease (yes vs. no), cancer (yes vs. no), hypertension (yes vs. no), diabetes mellitus (yes vs. no). IL-1 $\beta$, interleukin 1 beta; IL-2R, interleukin-2 receptor; IL-6, interleukin 6; IL-10, interleukin 10; TNF $\alpha$, tumor necrosis factor alpha. 
cytokine storm syndrome or cytokine release syndrome (CRS). Blocking IL-6 has been the focus in mitigating the cytokine storm-related symptoms in patients with severe COVID-19. Nevertheless, regarding the clinical symptoms of COVID-19, including lung injury and circulation of various cytokines, the cytokine profiles in patients with COVID-19 were distinct from those of the typical cytokine storm including CRS, hemophagocytic lymphohistiocytosis, or macrophage activation syndrome $(19,20)$. IL-6 antagonists, which have been approved for treating chimeric antigen receptor $\mathrm{T}$ cell-induced CRS, have demonstrated various effects in patients with COVID-19 in multiple studies (21). Because of the varying cohorts and intervention criteria, it is difficult to draw any conclusions. As the most common adverse event, serious infections were additional concerns. Somers et al. found that tocilizumab is associated with an increased proportion of superinfections in mechanically ventilated patients with COVID-19 (22). It is thus important to determine who might benefit from these therapy regimes considering heterogeneity of patients with COVID-19. Rossotti et al. performed a retrospective, single-center analysis on 74 patients treated with tocilizumab and 148 matched controls (23). A steep increase in IL-6 levels in patients indicates a poor clinical outcome. Similarly, Quartuccio et al. showed that out of 24 patients who received tocilizumab treatment, 6 patients died. Further, $24-48 \mathrm{~h}$ post-tocilizumab treatment, IL-6 serum levels were significantly higher in non-survivors than those in survivors (24). Two studies found that low levels of IL-6 at the baseline were associated with response to sarilumab or tocilizumab treatment $(25,26)$. Taken together with our results, these findings suggest that supervising the dynamic changes in serum IL-6 levels might offer an opportunity to define the inclusion population, predict prognosis, and adjust the treatment regime in clinical trials or in off-label treatment with IL-6 antagonists.

We found that continuously elevated levels of serum IL-8 in non-survivors compared to the survivors despite statistical differences were only observed in patients after two weeks of hospitalization. IL-8 is normally secreted by multiple cells exposed to stimuli and is considered the primary molecule of acute inflammation. Increased neutrophils have been thought to correlate with the severity of patients with COVID-19 (27). IL-8 induces potent neutrophil chemotaxis and promotes angiogenesis, which might result in deterioration of COVID-19. IL-6 signaling promotes IL-8 recruitment and enhances the inflammation cascade.
We found that the increase in serum levels of IL10 , which is normally considered an anti-inflammatory molecule, correlated with an increase in the levels of pro-inflammatory factors including IL-6, and was also a predictor of survival in ICU patients regardless of other severity factors. SARS-CoV-2 invasion is a trigger that induces a broad range of immunological events. High levels of circulating pro-inflammatory cytokines lead to tissue damage and multi-organ failure. As a result, a negative feedback loop might be activated to alleviate the response and promote a transition from immune hyperactivity to the resolution phase. Studies have shown that anti-inflammatory and pro-inflammatory reactions arise concomitantly in patients with sepsis $(28,29)$. A shortage of IL-10 is indicated by sustained elevation of TNF, IL-12, and interferon gamma levels in infected mice (30). Therefore, increased levels of circulating IL-10 in patients with severe COVID-19 suggested that an immune regulatory network was activated to limit the magnitude of the immune response and repair the damage. The combination of pro-inflammatory and anti-inflammatory mediators represents the immunological pathogenesis of SARS-CoV-2 infection. Ying et al. reported that IL-6, IL-7, IL-10, IL-18, granulocyte colonystimulating factor, macrophage colony-stimulating factor, monocyte chemoattractant protein-1 (MCP-1), MCP-3, interferon gamma-induced protein 10 (IP-10), monokine induced by gamma (MIG), and macrophage inflammatory protein 1 alpha (MIP1 $\alpha)$ were associated with the severity of COVID-19 (31). In another study that enrolled patients with moderate and severe COVID-19, IL-6 and IL-10 were used as predictors for recognizing patients at high risk of deterioration (12).

To the best of our knowledge, this is the first study on the dynamic variation of specific cytokine profiles and its correlation with the outcome of patients with COVID-19 admitted to the ICU. The limitations of this single-center study include small sample size and missing laboratory parameters that may induce potential bias. Second, superimposed bacterial infections might affect serum levels of cytokines in some patients. Finally, we observed the survival of patients during their ICU stay, and this might differ slightly from the final clinical outcome.

\section{Conclusions}

Our results signified that circulating cytokine biomarkers might prove useful for evaluating therapeutic responses and for optimizing the therapeutic strategies in patients with 
severe COVID-19.

\section{Acknowledgments}

We thank all the patients and their families in this study. Funding: None.

\section{Footnote}

Reporting Checklist: The authors have completed the STROBE reporting checklist. Available at http://dx.doi. org/10.21037/apm-20-2134

Data Sharing Statement: Available at http://dx.doi. org/10.21037/apm-20-2134

Conflicts of Interest: All authors have completed the ICMJE uniform disclosure form (available at http://dx.doi. org/10.21037/apm-20-2134). The authors have no conflicts of interest to declare.

Ethical Statement: The authors are accountable for all aspects of the work in ensuring that questions related to the accuracy or integrity of any part of the work are appropriately investigated and resolved. The study was performed in accordance with the Declaration of Helsinki (as revised in 2013) and approved by the Ethics Committee of Shanghai General Hospital of Shanghai Jiao Tong University School of Medicine (No. 2020-29). Individual consent was waived for this retrospective study.

Open Access Statement: This is an Open Access article distributed in accordance with the Creative Commons Attribution-NonCommercial-NoDerivs 4.0 International License (CC BY-NC-ND 4.0), which permits the noncommercial replication and distribution of the article with the strict proviso that no changes or edits are made and the original work is properly cited (including links to both the formal publication through the relevant DOI and the license). See: https://creativecommons.org/licenses/by-nc-nd/4.0/.

\section{References}

1. Mehta P, McAuley DF, Brown M, et al. COVID-19: consider cytokine storm syndromes and immunosuppression. Lancet 2020;395:1033-4.

2. Merad M, Martin JC. Pathological inflammation in patients with COVID-19: a key role for monocytes and macrophages. Nat Rev Immunol 2020;20:355-62.

3. Wu C, Chen X, Cai Y, et al. Risk Factors Associated With Acute Respiratory Distress Syndrome and Death in Patients With Coronavirus Disease 2019 Pneumonia in Wuhan, China. JAMA Intern Med 2020;180:934-43.

4. Cavalli G, De Luca G, Campochiaro C, et al. Interleukin-1 blockade with high-dose anakinra in patients with COVID-19, acute respiratory distress syndrome, and hyperinflammation: a retrospective cohort study. Lancet Rheumatol 2020;2:e325-e331.

5. De Luca G, Cavalli G, Campochiaro C, et al. GMCSF blockade with mavrilimumab in severe COVID-19 pneumonia and systemic hyperinflammation: a singlecentre, prospective cohort study. Lancet Rheumatol 2020;2:e465-e473.

6. Eimer J, Vesterbacka J, Svensson AK, et al. Tocilizumab shortens time on mechanical ventilation and length of hospital stay in patients with severe COVID-19: a retrospective cohort study. J Intern Med 2021;289:434-6.

7. Iglesias-Julián E, López-Veloso M, de-la-Torre-Ferrera $\mathrm{N}$, et al. High dose subcutaneous Anakinra to treat acute respiratory distress syndrome secondary to cytokine storm syndrome among severely ill COVID-19 patients. J Autoimmun 2020;115:102537.

8. Potere N, Di Nisio M, Cibelli D, et al. Interleukin-6 receptor blockade with subcutaneous tocilizumab in severe COVID-19 pneumonia and hyperinflammation: a casecontrol study. Ann Rheum Dis 2021;80:1-2.

9. Sanders JM, Monogue ML, Jodlowski TZ, et al. Pharmacologic Treatments for Coronavirus Disease 2019 (COVID-19): A Review. JAMA 2020;323:1824-36.

10. Xu X, Han M, Li T, et al. Effective treatment of severe COVID-19 patients with tocilizumab. Proc Natl Acad Sci U S A 2020;117:10970-5.

11. Del Valle DM, Kim-Schulze S, Huang HH, et al. An inflammatory cytokine signature predicts COVID-19 severity and survival. Nat Med 2020;26:1636-43.

12. Han $\mathrm{H}, \mathrm{Ma} \mathrm{Q}, \mathrm{Li}$ C, et al. Profiling serum cytokines in COVID-19 patients reveals IL-6 and IL-10 are disease severity predictors. Emerg Microbes Infect 2020;9:1123-30.

13. Ferraccioli G. More evidences on which biologic and which pathway is key in severe-critical COVID-19 pneumonia. Ann Rheum Dis 2020. doi: 10.1136/ annrheumdis-2020-218523.

14. National Health Committee of the People's Republic of China (2020) Guideline for Diagnosis and Treatment of Novel Coronavirus Pneumonia (Trial Version Sixth) 
Available online: http://www.nhc.gov.cn/xcs/zhengcwj/202 002/8334a8326dd94d329df351d7da8aefc2.shtml

15. Wolff D, Nee S, Hickey NS, et al. Risk factors for Covid-19 severity and fatality: a structured literature review. Infection 2021;49:15-28.

16. Liang W, Liang H, Ou L, et al. Development and Validation of a Clinical Risk Score to Predict the Occurrence of Critical Illness in Hospitalized Patients With COVID-19. JAMA Intern Med 2020;180:1081-9.

17. Wu G, Yang P, Xie Y, et al. Development of a clinical decision support system for severity risk prediction and triage of COVID-19 patients at hospital admission: an international multicentre study. Eur Respir J 2020;56:2001104.

18. Chen R, Sang L, Jiang M, et al. Longitudinal hematologic and immunologic variations associated with the progression of COVID-19 patients in China. J Allergy Clin Immunol 2020;146:89-100.

19. Behrens EM, Koretzky GA. Review: Cytokine Storm Syndrome: Looking Toward the Precision Medicine Era. Arthritis Rheumatol 2017;69:1135-43.

20. Mangalmurti N, Hunter CA. Cytokine Storms: Understanding COVID-19. Immunity 2020;53:19-25.

21. Madenidou AV, Bukhari M. Real-life experience of tocilizumab use in COVID-19 patients. Rheumatology (Oxford) 2020;59:2163-6.

22. Somers EC, Eschenauer GA, Troost JP, et al. Tocilizumab for treatment of mechanically ventilated patients with COVID-19. Clin Infect Dis 2020. doi: 10.1101/2020.05.29.20117358.

23. Rossotti R, Travi G, Ughi N, et al. Safety and efficacy of anti-il6-receptor tocilizumab use in severe and critical patients affected by coronavirus disease 2019: A comparative analysis. J Infect 2020;81:e11-e17.

24. Quartuccio L, Sonaglia A, Pecori D, et al. Higher levels of IL-6 early after tocilizumab distinguish survivors from nonsurvivors in COVID-19 pneumonia: A possible indication for deeper targeting of IL-6. J Med Virol 2020;92:2852-6.

25. Montesarchio V, Parrela R, Iommelli C, et al. Outcomes and biomarker analyses among patients with COVID-19 treated with interleukin 6 (IL-6) receptor antagonist sarilumab at a single institution in Italy. J Immunother Cancer 2020;8:e01089.

26. Squillace N, Pozzi MR, Gustinetti G, et al. Therapy of Sars-Coronavirus-2 pneumonia: is there an optimal IL-6 cut-off for successful tocilizumab treatment? Clin Infect Dis 2020. doi: 10.1093/cid/ciaa1282.

27. Laforge M, Elbim C, Frère C, et al. Tissue damage from neutrophil-induced oxidative stress in COVID-19. Nat Rev Immunol 2020;20:515-6.

28. Osuchowski MF, Craciun F, Weixelbaumer KM, et al. Sepsis chronically in MARS: systemic cytokine responses are always mixed regardless of the outcome, magnitude, or phase of sepsis. J Immunol 2012;189:4648-56.

29. Osuchowski MF, Welch K, Siddiqui J, et al. Circulating cytokine/inhibitor profiles reshape the understanding of the SIRS/CARS continuum in sepsis and predict mortality. J Immunol 2006;177:1967-74.

30. Couper KN, Blount DG, Riley EM. IL-10: the master regulator of immunity to infection. J Immunol 2008;180:5771-7.

31. Chi Y, Ge Y, Wu B, et al. Serum Cytokine and Chemokine Profile in Relation to the Severity of Coronavirus Disease 2019 in China. J Infect Dis 2020;222:746-54.
Cite this article as: Li J, Rong L, Cui R, Feng J, Jin Y, Chen X, $\mathrm{Xu}$ R. Dynamic changes in serum IL-6, IL-8, and IL-10 predict the outcome of ICU patients with severe COVID-19. Ann Palliat Med 2021;10(4):3706-3714. doi: 10.21037/apm-20-2134 
Supplementary

Table S1 The co-variates included in the final model between cytokine levels and outcome in 40 patients with COVID-19 in ICU

\begin{tabular}{lccc}
\hline Variables & Survivors $(\mathrm{n}=29)$ & Non-survivors $(\mathrm{n}=11)$ & $\mathrm{P}$ value \\
\hline Age $(\mathrm{y})$ & 0 (ref) & $0.008(0.005,0.115)$ & $<0.001$ \\
Sex & 0 (ref) & $-0.083(-0.19,0.235)$ & 0.13 \\
Absolute lymphocyte count (per $\mathrm{mL})$ & 0 (ref) & $0.32(0.09,0.54)$ & 0.005 \\
Absolute neutrophils count (per $\mathrm{mL})$ & 0 (ref) & $-0.01(-0.31,0.12)$ & 0.37 \\
Direct bilirubin (mmol/L) & 0 (ref) & $0.60(0.43,0.78)$ & $<0.001$ \\
Lactate dehydrogenase (U/L) & 0 (ref) & $0.11(-0.14,0.36)$ & 0.39 \\
Blood Urea Nitrogen (mmol/L) & 0 (ref) & $-0.11(-0.33,0.11)$ & 0.32 \\
D-dimer (mg/L) & 0 (ref) & $0.29(-0.12,0.70)$ & 0.16 \\
C-reactive protein (mg/L) & 0 (ref) & $0.29(-0.12,0.70)$ & 0.16 \\
History of hypertension (yes vs. no) & 0 (ref) & $0.356(0.270,0.442)$ & $<0.001$ \\
History of diabetes mellitus (yes vs. no) & 0 (ref) & $0.048(-0.062,0.157)$ & 0.39 \\
History of COPD (yes vs. no) & 0 (ref) & $0.292(0.113,0.472)$ & 0.002 \\
History of cancer (yes vs. no) & 0 (ref) & $0.733(0.624,0.843)$ & $<0.001$ \\
\hline
\end{tabular}

